\title{
Spinfoam 2d quantum gravity and discrete bundles
}

\author{
Daniele Oriti ${ }^{a}$, Carlo Rovelli ${ }^{b c}$, Simone Speziale ${ }^{a c}$ \\ ${ }^{a}$ DAMTP, Centre for Mathematical Sciences, University of Cambridge CB3 oWA, Cambridge, EU. \\ ${ }^{b}$ Centre de Physique Théorique de Luminy, Université de la Méditerranée, F-13288 Marseille, EU. \\ ${ }^{c}$ Dipartimento di Fisica dell'Università "La Sapienza", INFN Sez. Roma1, I-00185 Roma, EU.
}

September 11, 2018

\begin{abstract}
In 4 dimensions, general relativity can be formulated as a constrained $B F$ theory; we show that the same is true in 2 dimensions. We describe a spinfoam quantization of this constrained $B F$-formulation of $2 \mathrm{~d}$ riemannian general relativity, obtained using the Barrett-Crane technique of imposing the constraint as a restriction on the representations summed over. We obtain the expected partition function, thus providing support for the viability of the technique. The result requires the nontrivial topology of the bundle where the gravitational connection is defined, to be taken into account. For this purpose, we study the definition of a principal bundle over a simplicial base space. The model sheds light also on several other features of spinfoam quantum gravity: the reality of the partition function, the geometrical interpretation of the Newton constant, and the issue of possible finiteness of the partition function of quantum general relativity.
\end{abstract}

\section{Introduction}

Spinfoam formulations of quantum gravity can be derived in several ways (for reviews see [1, 2, 3.) One technique is based on the relation between general relativity $(\mathrm{GR})$ and $B F$ theory: GR can be expressed as a $B F$ theory plus a constraint, called the "Plebanski constraint". BF theory has a well-understood covariant quantization, given by a Feynman-like sum over representations of the gauge group, associated with (faces of a two-complex interpreted as the two-skeleton of) a simplicial discretization of the spacetime manifold. A spinfoam model for quantum GR can be obtained enforcing the Plebanski constraint into the spinfoam sum and, eventually, summing over two-complexes. The constraint can be enforced as a restriction on the class of representations summed over. This way of enforcing the constraint is called the Barrett-Crane technique and the resulting model is called the Barrett-Crane model (although Barrett and Crane's original rationale for restricting the class of representations was different 4.) Variants of this model have been studied, both in the riemannian and the lorentzian context; some of these have remarkable finiteness properties [5, 6, 7] and, although many issues remain unclear, the spinfoam formalism is often regarded as the covariant version of loop quantum gravity [3, 8$]$ and as candidate for computing transition amplitudes in quantum gravity.

A source of concern about this approach is the legitimacy of the Barrett-Crane technique. Ideally, one would like to test it by analyzing the physics predicted by the model, but our understanding of background independent physics is still insufficient for this. In this situation, it is interesting to test whether the technique works in a context which is simple and under control. This is what we do in this paper.

Indeed, we show below that in two dimensions (2d), GR admits a formulation as a constrained $B F$ theory, analogous to the $4 \mathrm{~d}$ theory. Riemannian $2 \mathrm{~d}$ quantum GR has been studied with several techniques (see [9] and references therein) and its partition function is known. Quantum BF theory in 2d has been studied [10; its quantization using the spinfoam formalism and group field theory (GFT) techniques [11, 12] was considered in [13. It is therefore natural to study the spinfoam quantization of $2 \mathrm{~d}$ riemannian GR using the Barrett-Crane technique, in order to test such a technique. Here we do so and we find that the BarrettCrane technique works well in the $2 \mathrm{~d}$ context, and it yields the expected partition function. Remarkably, the partition function of $2 \mathrm{~d}$ quantum GR turns out to be finite and well-defined, in spite of the fact that the $B F$ partition function diverges; thus, the Barrett-Crane technique improves the finiteness of the spinfoam sum, in $2 \mathrm{~d}$. We will comment on the interpretation of this fact and on its relevance for the $4 \mathrm{~d}$ case. 
In spite of the simplicity of the $2 \mathrm{~d}$ model, the way the quantization works is rather nontrivial. In order for the spinfoam model to give the correct partition function, it is necessary to take the nontrivial topology of the principal bundle on which the $B F$ theory is defined (which is distinct from the topology of the base manifold, namely the topology of spacetime) into account. A naive quantization that disregards the bundle topology gives an incorrect result. This fact raises the question of whether similar aspects should be considered also in higher dimensions.

Now, spinfoam quantization requires a discretization of the spacetime manifold. The definition of a nontrivial principal bundle over a discrete base is not immediate. In the continuum, the nontriviality of the bundle is encoded in continuous transition functions on the overlaps between open sets covering the base; in a discrete principal bundle, suitable discrete data must encode the bundle topology. We thus need to define the geometry of such a discrete principal bundle, in order to construct the model. We do this in Section 4 and, in a different and more detailed manner, in the Appendix.

A second issue we need to address is the scaling of the connection, namely the "size" of the internal gauge group. It is convenient to distinguish between the group elements that define the spinfoam model and the parallel propagators of the gravitational connection. The scaling factor between the two is where the Planck constant and the Newton constant are hidden, in a spinfoam model.

With all this technology at hand, the quantization of $B F$ theory is straightforward, and the BarrettCrane technique yields the expected $2 \mathrm{~d}$ gravity partition function, with no divergences appearing. The spinfoam model naturally yields the real partition function, related to the cosinus of the action instead than its complex exponential, precisely as it happens for the 3d Ponzano-Regge model [14. The reason for this and the relation with the orientation of the manifold and time-inversion, are transparent in $2 \mathrm{~d}$. Thus the $2 \mathrm{~d}$ case sheds light and provides support for the spinfoam approach to quantum gravity.

In the next section we give a short review of $2 \mathrm{~d}$ gravity, we illustrate its relation with $B F$ theory and we point out the relevance of the bundle topology. In Section 3 we review the spinfoam quantization of $B F$ theory and we present the spinfoam quantization of $2 \mathrm{~d}$ GR in the case in which spacetime has the topology of the torus and the bundle is trivial. Section 4 develops the mathematical machinery to deal with nontrivial discretized bundles. In Section [5 we apply this machinery to 2d GR for a general topology, and we show that the Barrett-Crane technique yields a finite partition function, with the expected form. In section 6 we summarize our results, and we discuss the relevance of the issues here raised for the higher dimensional cases. In the Appendix, we develop a definition of discrete bundles which may be more appropriate to deal with higher dimensions and with the GFT formalism, and we suggest a possibility to include the data characterizing the bundle topology into the GFT formalism, by modifying the kinetic term of the GFT action.

\section{General Relativity as a constrained $B F$ theory in $2 \mathrm{~d}$}

\section{$2.12 \mathrm{~d}$ GR}

We consider riemannian general relativity in two dimensions. The theory is defined by the Einstein-Hilbert action

$$
S_{\mathrm{GR}}[g]=\frac{1}{16 \pi G} \int d^{2} x \sqrt{\operatorname{det} g} R
$$

for a 2 d riemannian metric field $g_{\mu \nu}(x), \mu, \nu=1,2$. Here $G$ is the Newton constant, $R$ the Ricci scalar and $\operatorname{det} g$ the determinant of $g_{\mu \nu}$. In $2 \mathrm{~d}$, the Riemann tensor has only one independent component, say $R_{1212}$; the Ricci tensor and the the Ricci scalar are determined by

$$
(\operatorname{det} g) R_{\mu \nu}=g_{\mu \nu} R_{1212}, \quad(\operatorname{det} g) R=2 R_{1212},
$$

and therefore the Einstein tensor vanishes identically

$$
R_{\mu \nu}-\frac{1}{2} g_{\mu \nu} R \equiv 0 .
$$

It follows that the vacuum Einstein equations are satisfied by any metric. In fact, the action (1) is invariant under local variations of the metric. In particular, on a compact $2 \mathrm{~d}$ surface $M$, the Gauss-Bonnet theorem (see for instance [15]) states that

$$
\int_{M} d^{2} x \sqrt{\operatorname{det} g} R=4 \pi \chi(M)
$$


where $\chi(M)$ is the Euler's characteristic of $M$, an integer that depends only on the genus $g$ of the manifold, namely the number of holes, via $\chi=2-2 g$. Hence on a compact manifold $M$

$$
S_{\mathrm{GR}}[g]=\frac{\chi(M)}{4 G} .
$$

Formally, the theory can be covariantly quantized by writing the partition function

$$
\tilde{Z}_{\mathrm{GR}}[M]=\int \mathcal{D} g e^{\frac{i}{\hbar} S_{\mathrm{GR}}[g]},
$$

where $\mathcal{D} g$ is an unknown measure on the infinite dimensional space of metrics modulo diffeomorphisms at fixed topology. We may assume that this measure is normalized, that is

$$
\int \mathcal{D} g=1
$$

Notice that the gravitational constant $G$ in $2 \mathrm{~d}$ has the inverse physical dimensions of an action, and that the product $\hbar G$ is dimensionless. Since the action does not depend on the particular choice of the metric, but only on the topology of the manifold, (5) gives

$$
\tilde{Z}_{\mathrm{GR}}[M]=\int \mathcal{D} g e^{\frac{i \chi(M)}{4 \hbar G}}=e^{\frac{i \chi(M)}{4 \hbar G}} \int \mathcal{D} g
$$

Using the assumption (7) that $\mathcal{D} g$ is normalized, we obtain the following partition function for $2 \mathrm{~d}$ riemannian general relativity

$$
\tilde{Z}_{\mathrm{GR}}[M]=e^{\frac{i \chi(M)}{4 \hbar G}} .
$$

In a background dependent quantum theory, the sign of the exponent in (6) is determined by time orientation: it is reversed under $t \rightarrow-t$. The partition function and all transition amplitudes go into their complex conjugates under time reversal. The situation in a background independent theory is less clear. It is often argued that both directions of time evolution, or, equivalently, both orientations of the manifold $M$, must be summed over in the Feynman integral. This yields a real partition function, and real transition amplitudes, and is consistent with the fact that the Wheeler-DeWitt equation has real solutions. See [3] and [16] for a more detailed discussion. Here, we consider also the real partition function

$$
Z_{\mathrm{GR}}[M]=\int \mathcal{D} g\left(e^{\frac{i}{\hbar} S_{\mathrm{GR}}[g]}+e^{-\frac{i}{\hbar} S_{\mathrm{GR}}[g]}\right)=2 \cos \left(\frac{\chi(M)}{4 \hbar G}\right),
$$

which is (twice) the real part of (9). Our task is to recover this result from a spinfoam quantization of the theory, without using an ill-defined infinite-dimensional measure, and without encountering any divergence.

We use the "dyad" formalism, namely the $2 \mathrm{~d}$ analog to the tetrad formalism. Let $e^{i}(x)=e_{\mu}^{i}(x) d x^{\mu}, i=$ 1,2 , be a dyad one-form field (with $\operatorname{det} e \neq 0$ ) related to the metric by $g_{\mu \nu}(x)=e_{\mu}^{i}(x) e_{\nu}^{j}(x) \delta_{i j}$. Let $\omega^{i}{ }_{j}(x)=\omega_{\mu j}^{i}(x) d x^{\mu}$ be the corresponding spin-connection one-form, defined by the Cartan structure equation

$$
d e^{i}+\omega^{i}{ }_{j} \wedge e^{j}=0 .
$$

$\omega^{i}{ }_{j}$ is an abelian $S O(2)$ connection on the frame bundle. Its curvature is the Lie-algebra valued two-form $F^{i}{ }_{j}=\frac{1}{2} F^{i}{ }_{j \mu \nu} d x^{\mu} \wedge d x^{\nu}$ defined by $F^{i}{ }_{j}=d \omega^{i}{ }_{j}$. Using the generator $\tau$ of the so(2) algebra, with components

$$
\tau^{i}{ }_{j}=\left(\begin{array}{cc}
0 & 1 \\
-1 & 0
\end{array}\right)
$$

we can write $\omega^{i}{ }_{j}=\omega \tau^{i}{ }_{j}$ where $\omega$ is a real valued one-form; and $F^{i}{ }_{j}=f \tau^{i}{ }_{j}$ where $f=d \omega$ is a real valued two-form. We shall denote $s o(2)$ matrices with capital letters, thus $F=f \tau$ has matrix components $f \tau^{i}{ }_{j}$, and so on.

The curvature of the spin connection, $F$, is related to the Riemann curvature by $e_{\mu}^{i} R^{\mu}{ }_{\nu \rho \sigma}=e_{\nu}^{j} F_{j \rho \sigma}^{i}$. Using this, it is easy to see that in terms of these fields the action (11) reads

$$
S_{\mathrm{GR}}[e]=\frac{1}{8 \pi G} \int \operatorname{sgn}(e) f=\frac{\operatorname{sgn}(e)}{8 \pi G} \int d \omega,
$$


where $\operatorname{sgn}(e)$ is the sign of the determinant of $e_{\mu}^{i}$. Because of Stokes theorem, this action over an open set is a boundary term and is therefore invariant under local field variations. Hence any dyad field, or any connection $\omega$ solves the equations of motion locally.

Notice that if we formally define the quantum theory in terms in this formalism

$$
Z_{\mathrm{GR}}[M]=\int \mathcal{D} e e^{\frac{i}{\hbar} S_{\mathrm{GR}}[e]},
$$

we must sum over both signs of det $e$ and therefore we obtain the real partition function (10). Clearly, a dyad with negative determinant, such as $e^{1}=-d x^{1}, e^{2}=d x^{2}$, is equivalent to a "time-reversed" field, or an opposite orientation of $M$. Therefore the dyad formalism leads naturally to the real partiction function. This can be compared with the case the Ponzano-Regge theory, where the same phenomenon happens.

\subsection{Constrained $B F$ theory}

We now show that $2 \mathrm{~d}$ riemannian GR can be expressed as a constrained $B F$, like $4 \mathrm{~d}$ GR. Let $\omega$ be a $S O(2)$ connection and $B=b \tau$ a scalar field with values in the $s o(2)$ algebra. We consider the $B F$ theory defined by the action

$$
S_{B F}[\omega, B]=-\frac{1}{2} k \int \operatorname{tr}[B F]=k \int b f
$$

where $f=d \omega$. Here $k$ a constant with the physical dimensions of an action. ${ }^{1}$ The factor $-1 / 2$ comes from $\operatorname{tr}[\tau \tau]=-2$. The equations of motion are the standard $B F$ equations

$$
d B(x)=0
$$

and

$$
F(x)=0,
$$

which are solved by any flat $\omega$ and constant $B$. The theory is topological, like 2d GR, namely it has no local degrees of freedom, but is different from $2 \mathrm{~d}$ GR. In fact, we can identify the connection $\omega$ of the $B F$ theory with the spin connection $\omega$ of GR, but while the equations of motion of $2 \mathrm{~d}$ GR are solved by any $\omega$, the equations of motion of $B F$ are solved only by flat connections. Hence GR and $B F$ are distinct theories in $2 \mathrm{~d}$, as they are distinct theories in $4 \mathrm{~d}$.

Recall that in $4 \mathrm{~d}$ GR can be obtained from $B F$ theory by adding a constraint. Schematically, the action of $4 \mathrm{~d}$ GR can be written as

$$
S_{\mathrm{GR}}[e, \omega]=\frac{1}{16 \pi G} \int \operatorname{tr}[*(e \wedge e) \wedge F]
$$

where $e$ is the (Minkowski valued) tetrad (one-form) field, $\omega$ is the corresponding spin connection, $F$ its curvature and the star indicates the Hodge dual. We can add a constraint to a $S O(4) B F$ action, as follows

$$
S_{\mathrm{GR}}[B, \omega, \lambda]=k \int \operatorname{tr}[B \wedge F-\lambda B \wedge B] .
$$

Varying the Lagrange multiplier $\lambda$ we obtain a Plebanski constraint of the form $B \wedge B=0$, which is solved when $B$ has the form $B=*(e \wedge e)$. Inserting this back into action, and identifying $k$ with $1 / 16 \pi G$ gives the GR action. See [17, 18, for details.

Let us now see how the same can be obtained in $2 \mathrm{~d}$. Consider a modification of the $B F$ action obtained adding a constraint by means of a two-form lagrangian multiplier $\lambda$ as follows

$$
S_{\mathrm{GR}}[B, \omega, \lambda]=-\frac{1}{2} k \int \operatorname{tr}[B F-\lambda(B B+\mathbf{1})]=k \int\left[b f-\lambda\left(b^{2}-1\right)\right] .
$$

The equations of motion obtained varying $\omega, B, \lambda$ are, respectively,

$$
\begin{aligned}
d B(x) & =0, \\
F(x) & =2 \lambda(x) \tau, \\
-\frac{1}{2} \operatorname{tr}[B B] & =1 .
\end{aligned}
$$

\footnotetext{
${ }^{1}$ Using dimensionless coordinates, the covariant derivative, the connection and the curvature are dimensionless.
} 
Eq (21) follows from (23). Equation (22) shows that the connection $\omega$ is free to take any value, because $\lambda(x)$ is arbitrary, precisely as in 2d GR. Equation (23) is the 2d Plebanski constraint. Inserting it back in the action, and choosing the value

$$
k=\frac{1}{8 \pi G}
$$

for the coupling constant, we obtain the GR action (13). Recall that in $4 \mathrm{~d}$ the addition of a constraint frees degrees of freedom, because the new constraint constrains a constrainer: the Lagrange multiplier $B$. Here, something similar happens: the constraint enlarges the spaces of classical solutions of the the equation of motion; see (22). However, this does not increase the number of degrees of freedom because of gauge equivalence. The difference is that in $4 \mathrm{~d}$ the temporal components of the $B$ field are Lagrange multipliers, while in $2 \mathrm{~d}$ the $B$ field is simply the conjugate variable to the spatial component of $\omega$, thus the Plebanski constraint (23) does not constrain a multiplier, and thus does not add degrees of freedom to the theory. ${ }^{2}$

Notice that the Plebanski constraint (23) has two solution: $B= \pm \tau$, corresponding to the two possible signs of $\operatorname{det} e$. The $2 \mathrm{~d}$ theory mimics also the multiplicity of the solutions of the Plebanski constraint that arises in $4 \mathrm{~d}$ [16, 17].

In $2 \mathrm{~d}$ the situation is far simpler than in $4 \mathrm{~d}$. The gauge group is abelian and the theory is topological. Precisely because of this simplicity, we are interested in checking whether the Barrett-Crane technique works in this context. Thus, we want to quantize 2d GR following the Barrett-Crane strategy: we write the spinfoam quantization of the unconstrained BF model, and then impose the Plebanski constraint (23) as a restriction on the sum over representations. Before doing so, however, a crucial remark is necessary, presented in the following section.

\subsection{Topology of the frame bundle}

Let us return to the case of a compact $2 \mathrm{~d}$ space $M$. Notice that at first sight there seems to be a contradiction between (4) and (13): consider the action (13) for a compact manifold $M$. One could expect the Stokes theorem to imply that $S_{\mathrm{GR}}$ vanishes, since

$$
\int_{M} d \omega=\oint_{\partial M} \omega
$$

which vanishes if $M$ is compact and $\partial M=0$. But $S_{\mathrm{GR}}$ is equal to the right hand side of (4), which in general is nonvanishing. How could this be? The answer is that for a generic topology of the manifold we cannot define the dyad field $e^{i}(x)$ globally. For instance, there is no continuous nonvanishing 1-form $e^{1}(x)$ on a sphere. Thus we must define separate dyad fields $e_{a}$ on each local chart $a$. (Here and below $a, b, \ldots$ are not indices: they are subscripts labeling the chart in which the field $e_{a}$ is defined.) These must be related by a $S O(2)$ rotation $t_{a b}(x)=e^{\psi_{a b}(x) \tau}$ on the overlaps between charts

$$
e_{a}=t_{a b} e_{b}
$$

In turn, the spin connection one-form is defined only within local charts, where we call it $\omega_{a}$, and has gauge transformations

$$
\omega_{b}=\omega_{a}+d \psi_{a b} .
$$

on the overlaps. Equation (25) is correct only if $e$, and hence $\omega$, can be defined on the entire $M$. The only compact 2d topology where $e^{i}(x)$ can be defined globally is the torus, for which (25) is correct; but for the torus (25) is not in contradiction with (4), since the Euler characteristic $\chi$ of the torus is zero.

Consider an arbitrary principal bundle $P(M, S O(2))$ with $2 \mathrm{~d}$ base manifold $M$ and structure group $S O(2)$. Let $\omega$ be a connection on this bundle and $f$ its curvature. To compute the left hand side of (25) using Stokes theorem, we must first break the integral over $M$ into a sum of integrals over regions $a$, forming a partition of $M$, and each defined within a single chart. In each of these regions the connection $\omega$ is represented by a one-form $\omega_{a}$ and we can apply Stokes theorem. This gives

$$
\int_{M} f=\sum_{a} \int_{a} f=\sum_{a} \int_{a} d \omega_{a}=\sum_{a} \oint_{\partial a} \omega_{a}
$$

\footnotetext{
${ }^{2}$ We thank our referees for pointing this out.
} 
If we call $e_{a b}$ the boundary separating the regions $a$ and $b$, with an assigned orientation (say towards the right crossing from $a$ to $b$ ), this becomes

$$
\int_{M} f=\sum_{a b} \int_{e_{a b}} \omega_{a}=\sum_{(a b)} \int_{e_{a b}}\left(\omega_{a}-\omega_{b}\right)=\sum_{(a b)} \int_{e_{a b}} d \psi_{a b},
$$

where $\psi_{a b}$ is the transition function from the chart in which the region $a$ is contained to the chart in which the region $b$ is contained and -here and below- the notation $(a b)$ indicates unordered pairs. Notice that signs are well defined since $t_{a b}=t_{b a}^{-1}$ implies $\psi_{a b}=-\psi_{b a}$ and therefore

$$
\int_{e_{a b}} d \psi_{a b}=\int_{e_{b a}} d \psi_{b a} .
$$

Equation (29) shows that the integral of the curvature over the manifold is independent from the connection, but it depends on the topology of the bundle, which is coded in the transition functions $t_{a b}$. In fact, (22) is ( $2 \pi$ times) the the Euler number, a well-known topological invariant that characterizes the topology of the bundle $P=P(M, S O(2))$,

$$
e(P)=\frac{1}{2 \pi} \int_{M} f .
$$

The Euler number $e(P)$ is the integral of the first Euler class, ${ }^{3}$ which here is just $f$, over the base manifold. The topology of the bundle $P$ should not be confused with the topology of the base $M$ : there exist many topologically distinct bundles $P$ for each base space $M$. We illustrate this in more detail and give an explicit example in Section 4.1 It can be shown that the topology of an $S O(2)$ principal bundle over a $2 \mathrm{~d}$ base space $M$ is entirely characterized by the Euler number (31).

Let us now return to GR and come to the key point. In the case of GR, the gravitational spin connection $\omega$ is a connection on a principal bundle $P(M, S O(2))$ over spacetime $M$. However, this is not an arbitrary bundle: it is (isomorphic to) the frame bundle. This is because $\omega$ must satisfy the Cartan equation (11); $\omega$ is determined by the dyad field $e$, which defines a local linear map (a "soldering") between the associated $R^{2}$ vector bundle on which $\omega$ acts and the tangent bundle. This map is smooth and therefore the bundle on which $\omega$ acts is isomorphic to the tangent bundle, which in general has nontrivial topology. Thus, $\omega$ acts on (a bundle isomorphic to) the tangent bundle, and therefore it lives in the principal bundle of the local rotations of the tangent bundle, namely the frame bundle. We denote the frame bundle $P_{\mathrm{fr}}(M, S O(2))$, or simply $P_{\mathrm{fr}}$. The topology of the frame bundle is obviously determined by the topology of $M$ and in general is nontrivial. Now, in 2d it is well known, and it follows from (4), (13) and (31), that the frame bundle is the $P(M, S O(2))$ principal bundle whose Euler number (31) is equal to the Euler characteristic of the base space $M$. That is

$$
e\left(P_{\mathrm{fr}}\right)=\chi(M) .
$$

A priori, $B F$ theory can defined over an arbitrary principal bundle; but if we want to view GR as a modified $B F$ theory, the field $\omega$ of the $B F$ theory must be a connection on a bundle with the same topology as $P_{\text {fr }}$, namely satisfying (32). ${ }^{4}$ In applying the spinfoam quantization procedure, we have to be sure that the non-triviality of the bundle is taken into account, and to choose transition functions $t_{a b}$ satisfying (32). This consideration turns out to play a crucial role in the quantization.

There is one case which is particularly simple. This is the case of genus 1 , namely the torus $T_{2}$. The tangent bundle of the torus is trivial: $T\left(T_{2}\right)$ is homeomorphic to $T_{2} \times R^{2}$, and the frame bundle is correspondingly trivial: $P_{\mathrm{fr}}\left(T_{2}, S O(2)\right)$ is homeomorphic to $T_{2} \times S O(2)$. In fact, we can obviously choose a global dyad field on the torus, and hence a global spin connection one-form $\omega$. Equivalently, the torus admits a flat metric, hence the curvature can vanish everywhere, and the Euler characteristic $\chi\left(T_{2}\right)$ is zero.

Accordingly, we separate our analysis of the spinfoam formulation of $2 \mathrm{~d}$ GR into two steps. In the following section we discuss the case in wich the bundle is trivial, where all subtleties related to the global topology of the frame bundle are absent. This case is relevant for GR only when the topology of spacetime is a torus. In this case, we can show easily that the Barrett-Crane technique works and yields a finite

\footnotetext{
${ }^{3}$ In the literature the name of the class usually depends on the structure group of the bundle; Chern class is used for bundles with structure group $S U(N)$, and Euler class for bundles with structure group $S O(N)$. See for instance [23].

${ }^{4}$ In $2 \mathrm{~d}$, the soldering form $e$ has disappeared from the dynamics, since the action 13 depends only on $\omega$ and a global sign. However, its "ghost" remains, since the vector bundle on which the spin connection acts and the tangent bundle must have same topology: we can say that they are "soldered topologically."
} 


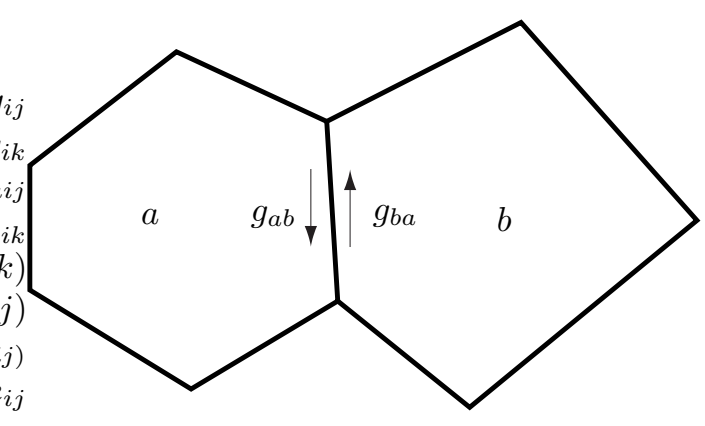

Figure 1: Two faces $a$ and $b$ of $\Delta^{*}$ and the group elements $g_{a b}$ and $g_{b a}$ on the edge $e_{a b}$ between the two.

and correct partition function. This result alone, however, is not very enlightening, since (10) gives simply $Z_{\mathrm{GR}}=2$ for the torus. Then in Section 4 we discuss the general case, taking the topology into account, and in Section [5 we derive equation (10) in the general case.

\section{Trivial principal bundle}

\subsection{Spinfoam quantization of $B F$}

Let us recall the standard spinfoam quantization of the $B F$ action (15). Formally, the quantum theory is defined by the partition function

$$
Z_{B F}=\int \mathcal{D} B \mathcal{D} \omega e^{i \frac{k}{\hbar} \int_{M} \operatorname{tr}[B F]} .
$$

In order to give meaning to this integral, we define a lattice version of the theory on a discrete manifold. Since the theory is topological, the discretization does not cut off physical degrees of freedom. Fix a triangulation $\Delta$ of the manifold. The triangulation consists of triangles (2-simplices) meeting at segments (1-simplices), in turn meeting in points (0-simplices); it is oriented, with orientation induced by $M$. Intuitively, we can view the triangles as flat, with the curvature concentrated on the 0-simplices, as in simplicial Regge geometry [19. We work with the cellular complex $\Delta^{*}$, dual to the triangulation $\Delta$. The dual cellular complex $\Delta^{*}$ is formed by faces $a, b, c \ldots$ (dual to the 0 -simplices). The two faces $a$ and $b$ meet along an edge that we call $e_{a b}$. Edges are dual to the 1-simplices. We orient $e_{a b}$ following the perimeter of $a$ clockwise. Therefore $e_{b a}$ is the same edge as $e_{a b}$, but with the opposite orientation. Edges, in turn, meet at trivalent vertices $v_{a b c}$, which are dual to the triangles of $\Delta$.

We replace the continuous fields $\omega$ and $B$ with discrete fields on $\Delta^{*}$, as follows. The connection $\omega$ is replaced by a group element $g_{a b}$ associated to each edge $e_{a b}$. See Fig 1 This is interpreted as the exponential of the one-form field $\frac{k}{\hbar} \omega$, the "scaled connection", along the edge

$$
g_{a b}=e^{\frac{k}{\hbar} \int_{e_{a b}} \omega} .
$$

Notice that this is not the parallel transport of the metric connection, which is given by $e^{\int_{e_{a b}} \omega}$. We shall comment on the meaning of this choice at the end of Section [5. We parametrize $S O(2)$ as

$$
g_{a b} \equiv e^{\phi_{a b} \tau}
$$

where $\phi_{a b} \in[0,2 \pi]$. Since $e_{b a}$ is $e_{b a}$ with the opposite orientation, we demand that

$$
g_{a b} g_{b a}=1 ;
$$

that is

$$
\phi_{a b}+\phi_{b a}=0 .
$$

The field $B$ is replaced by a variable $B_{a}=b_{a} \tau$ associated to each face $a$. The dynamical variables of the lattice theory are therefore $\left(g_{a b}, B_{a}\right)$, where $g_{a b} \in S O(2), B_{a} \in s o(2)$ and $g_{a b}$ and $g_{b a}$ are related by (36). 
We now define a group element $g_{a}$ related to the curvature on a face $a$. If the face $a$ is bounded by the faces $b_{1} \ldots b_{m}$, we define

$$
g_{a}=e^{\phi_{a} \tau} \equiv g_{a b_{1}} \ldots g_{a b_{m}}=e^{\left(\phi_{a b_{1}}+\ldots+\phi_{a b_{m}}\right) \tau},
$$

where $\phi_{a} \in[0,2 \pi)$. Notice that curvature lives on the faces, which are dual to the 0 -simplices of the original triangulation $\Delta$, which is where we expected curvature to live. We say that a discretized connection $g_{a b}$ is flat on $a$ if $g_{a}=\mathbf{1}$. It is convenient to introduce also the square root of $g_{a}$, defined by ${ }^{5}$

$$
g_{a}^{1 / 2} \equiv e^{\left(\phi_{a} / 2\right) \tau}
$$

Since we have parametrized the group elements with $\phi_{a} \in[0,2 \pi)$ the last equality defines a square root uniquely.

In the limit in which the triangulation is fine and the face $a$ has area $d^{2} x$, this satisfies

$$
g_{a}^{1 / 2}=\mathbf{1}+\frac{1}{2} f_{a} \tau d^{2} x
$$

The dynamics of the discrete model is defined by discretizing the action (15) as

$$
S_{B F}\left[B_{a}, g_{a b}\right]=-\hbar \sum_{a} \operatorname{tr}\left[B_{a} g_{a}^{1 / 2}\right]
$$

The reason for which it is convenient to use the square root is technical: it allows us to avoid the doubling of the degrees of freedom introduced by the discretization, as we shall see in a moment. An alternative technique for dealing with this problem was developed by Freidel and Louapre in [21].

This action approximates the continuous action in the limit of fine triangulations and reproduces the symmetries of the continuous action (see [20] for the $3 \mathrm{~d}$ case.) The action is invariant under local gauge transformations generated by a gauge parameter $\lambda_{a b c}$, which sits on vertices $v_{a b c}$. The discretized connection transforms as

$$
g_{a b} \rightarrow \lambda_{a b c} g_{a b} \lambda_{a b d}^{-1},
$$

where $v_{a b c}$ and $v_{a b d}$ are the vertices where the edge $e_{a b}$ begins and ends.

We can now give a precise definition to the formal functional integral (33) as

$$
Z_{B F}=\int \prod_{(a b)} d g_{(a b)} \prod_{a} d B_{a} e^{\frac{i}{\hbar} S\left[B_{a}, g_{a b}\right]}=\int \prod_{(a b)} d g_{(a b)} \prod_{a} d B_{a} e^{-i \sum_{a} \operatorname{tr}\left[B_{a} g_{a}^{1 / 2}\right]} .
$$

Here

$$
d g_{(a b)}=d g_{a b} d g_{b a} \delta\left(g_{a b} g_{b a}\right)
$$

and $d g_{a b}$ is the Haar measure of $S O(2)$. This is just the normalized measure of an angle, $d g_{a b} \equiv d \phi_{a b} / 2 \pi$. We take for $d B_{a}$ the Lebesgue measure on the $s o(2)$ algebra, that is $d B_{a} \equiv d b_{a}$. This defines quantum $B F$ theory. Let us now compute the partition function explicitly.

The integrals over $d B_{a}$ can be easily performed. Using

$$
-\operatorname{tr}[B g]=-\operatorname{tr}\left[b\left(\begin{array}{cc}
0 & 1 \\
-1 & 0
\end{array}\right)\left(\begin{array}{cc}
\cos \phi & \sin \phi \\
-\sin \phi & \cos \phi
\end{array}\right)\right]=2 b \sin \phi
$$

we have

$$
\int d B_{a} e^{-i \operatorname{tr}\left[B_{a} g_{a}^{1 / 2}\right]}=\int d b_{a} e^{2 i b_{a} \sin \left(\phi_{a} / 2\right)}=2 \pi \delta\left(2 \sin \left(\phi_{a} / 2\right)\right)=2 \pi \delta\left(\phi_{a}\right)=\delta\left(g_{a}\right)
$$

where $\delta(g)$ is the delta function on the group, defined with the Haar measure. (Had we used $g_{a}$ instead of $g_{a}^{1 / 2}$ in the action, we would have obtained $2 \pi \delta\left(\sin \phi_{a}\right)=2 \pi\left(\delta\left(\phi_{a}\right)+\delta\left(\phi_{a}-\pi\right)\right)=\delta\left(g_{a}\right)+\delta(-g)$. See [21] for an alternative solution to the same problem.) Using this, we obtain,

$$
Z_{B F}=\int \prod_{(a b)} d g_{(a b)} \prod_{a} \delta\left(g_{a}\right),
$$

\footnotetext{
${ }^{5}$ From the aesthetic point of view, this is a bit odd, also because we could take any power $g^{a}, 0<a<1$ of the group element. We thank our referees for pointing this out.
} 
which is the standard expression for the partition function of the $B F$ models.

The spinfoam sum is obtained using the Plancherel expansion the $\delta$ function on the group in irreducible representations. In our simple abelian case the $S O(2)$ irreducible representations are labelled by an integer $n$; they are one-dimensional and their character is $\chi^{(n)}(g)=e^{i n \phi}$. In other words, the Plancherel decomposition is here the standard Fourier expansion of the delta function

$$
\delta(\phi)=\frac{1}{2 \pi} \sum_{n} e^{i n \phi}
$$

Using this, we can write

$$
Z_{B F}=\int \prod_{(a b)} \frac{d \phi_{(a b)}}{2 \pi} \prod_{a} \sum_{n} e^{i n \phi_{a}} .
$$

We can exchange the sum with the integrals by writing this expression as a sum over all the possible assignments $\left\{n_{a}\right\}$ of a representation $n_{a}$ to each face $a$

$$
Z=\sum_{\left\{n_{a}\right\}} \int \prod_{(a b)} \frac{d \phi_{(a b)}}{2 \pi} e^{i \sum_{a} n_{a} \phi_{a}} .
$$

Each edge bounds exactly two faces. Using (38), this becomes

$$
Z=\sum_{\left\{n_{a}\right\}} \int \prod_{(a b)} \frac{d \phi_{(a b)}}{2 \pi} e^{i \sum_{a b} n_{a} \phi_{a b}} .
$$

Using (37), this becomes

$$
Z=\sum_{\left\{n_{a}\right\}} \int \prod_{(a b)} \frac{d \phi_{(a b)}}{2 \pi} e^{i \sum_{(a b)}\left(n_{a}-n_{b}\right) \phi_{a b}},
$$

where the sum is over unordered pairs. All integrals decouple. They can be performed using the abelian equivalent of the formula for the orthogonality of characters

$$
\int_{0}^{2 \pi} \frac{d \phi}{2 \pi} e^{-i n_{a} \phi} e^{i n_{b} \phi}=\delta_{n_{a} n_{b}} .
$$

The multiple sum reduces to a single sum of the identity over one index only,

$$
Z_{B F}=\sum_{n} 1
$$

Thus the partition function diverges badly, as does the partition function of $B F$ theory in $4 \mathrm{~d}$. Intuitively, this divergence is due to the infinite volume of the original algebra-valued variable $B(x)$, or equivalently by the presence of a translational gauge symmetry $B \rightarrow B+c$ for any constant field $c$, and could formally be eliminated by dividing $Z$ by the infinite volume of the algebra 20. As we shall see, this divergence disappears in the GR case, and therefore we do not worry about it here. On the other hand, the partition function is formally triangulation independent, as we expect from a topological quantum field theory with no local degrees of freedom.

The sum (54) is an extremely simplified spinfoam model: it is defined on a 2-complex, where faces are colored by representations of the gauge group. The "abelian Clebsch-Gordon conditions" force representations to match across each edge, hence they force all representations to be equal. The partition function of the model is then given by the weighted sum over all allowed colorings. The weight of each coloring is 1 for all representations.

\subsection{Quantum GR on the torus}

Let us now come to GR. As mentioned in the introduction, our strategy is to view GR as a $B F$ theory plus the additional Plebanski constraint (23), and to search for its quantum theory by enforcing this constraint 
into the spinfoam sum that defines quantum $B F$ theory. In order to do so, we have to identify the quantity representing the field $B$ in the spinfoam sum.

As in $4 \mathrm{~d}$, it is the representation $n_{a}$ associated to the face $a$ that represents the field $B_{a}$ on the face. Indeed, observe that in equation (46) we have integrated over $B_{a}$ to get the delta function; and then in equation (49) we have expanded back the delta function as a sum over $n_{a}$. That is, we have used the fact that

$$
\int d b_{a} e^{i b_{a} \phi_{a}}=2 \pi \delta\left(\phi_{a}\right)=\sum_{n_{a}} e^{i n_{a} \phi_{a}} .
$$

The equality is correct because the delta is defined on a compact domain: $\phi_{a} \in[0,2 \pi]$. (In fact, this equality expresses the reason for which a physical quantity conjugate to a variable living in a compact domain is quantized.) The discrete variable $n_{a}$ is therefore the quantized version of the continuous variable $B_{a}$. Hence, we translate the classical constraint on $B_{a}$ into a constraint on $n_{a}$. That is, we implement the Plebanski constraint (23) as a restriction on the representations summed over, as in done in the BarrettCrane models. We can mimic exactly the $4 \mathrm{~d}$ procedure by identifying the continuous field $B \in s o(2)$ with the $s o(2)$ generator $J$, as we identify angular momentum with the generator of the rotation group. Consider the quadratic Casimir $C=-\frac{1}{2} \operatorname{tr}[J J]$. Under the identification $B \leftrightarrow J$ the Plebanski constraint (23) reads

$$
C=-\frac{1}{2} \operatorname{tr}[J J]=-\frac{1}{2} \operatorname{tr}[B B]=1 .
$$

In the representation $n$, the generator $J$ is the matrix $n \tau$ (we are still working with representations of the algebra over the real numbers, with the generator given by the expression (12)) and the Casimir $C$ takes the value

$$
C=-\frac{1}{2} \operatorname{tr}[n \tau n \tau]=n^{2} .
$$

Hence the Plebanski constraint becomes

$$
n^{2}=1 \text {. }
$$

We enforce the Plebanski constraint (23) by restricting the spinfoam sum (51) to the representations satisfying (58). Then the infinite sum reduces to two terms, giving

$$
Z_{\mathrm{GR}}\left[T_{2}\right]=\sum_{n} \delta_{n^{2}, 1}=2 .
$$

where we have indicated explicitly that the theory is defined on a torus.

First, notice that the model becomes finite. At the end of the previous section we mentioned the fact that the symmetry under translations of the $B$ field is responsible for the divergence of the partition function. The Plebanski constraint (23) fixes this gauge, yielding a finite partition function.

Notice that also in $4 \mathrm{~d}$ the Plebanski constraint fixes part of the gauge symmetry, since the gauge symmetry group of $B F$ theory is larger than the one of GR. The interpretation of the the divergences of $4 \mathrm{~d}$ spinfoam gravity theory, however, is controversial. Some models are most probaly finite [5, 6, 7] (see [2, 3]); for these, the analogy with the $2 \mathrm{~d}$ case presented here is complete. But it has also been argued that the remaining divergences, that appear in other $4 \mathrm{~d}$ spinfoam gravity models, are to be expected, because they reflect the rest of the gauge invariance (see [20] for the relevant analysis in the $3 \mathrm{~d}$ case). If so, an extra gauge fixing has to be performed to obtain finite results. We do not enter in this debate here. In any case, the simple model discussed here illustrates how full gauge fixing is the condition for finiteness.

Second, at first sight the result $Z_{\mathrm{GR}}\left[T_{2}\right]=2$ may seem trivial, but notice that it agrees precisely with (10), for the following reason: we have assumed the bundle to be trivial, but in GR the bundle must be the frame bundle. The only case in which the frame bundle is trivial is the torus, for which $e\left(P_{\mathrm{fr}}\right)=\chi\left(T_{2}\right)=0$. Thus, the result we obtain is only valid for the torus, and therefore it agrees with (10). The result is therefore finite and correct.

However, to be convinced that this works in the general case we have to deal with arbitrary bundle topologies. For this, we need to define topologically nontrivial simplicial bundles; this is what we do in the following section. 


\section{Nontrivial bundle topology}

Our aim is now to give a useful definition of a discrete $2 \mathrm{~d}$ bundle, that is, a bundle over a cellular complex, defined with discrete topological data. This can be done in several ways; here we discuss a definition that allows a simple discretization of a field theory on a nontrivial continuous bundle. To motivate our definitions, we proceed by constructing the discrete bundle starting from an actual discretization of the continuous one. In the Appendix we present a different and more detailed construction, which should turn out to better match with the GFT formulation of the theory and to be more appropriate to the extension to higher dimensions.

\subsection{Bundles}

Let us begin by recalling some simple notions of differential geometry. A principal bundle $P(M, G)$, where $G$ is a Lie group and $M$ is a compact manifold, can be uniquely reconstructed (as an equivalence class of coordinate bundles) by covering $M$ with topologically trivial open subsets $U_{a}$ and giving a set of transition functions

$$
t_{a b}: U_{a} \cap U_{b} \rightarrow G .
$$

The transition functions must to satisfy three conditions:

$$
\begin{aligned}
t_{a a}(x) & =\mathbf{1}, \\
t_{a b}(x) t_{b a}(x) & =\mathbf{1}, \\
t_{a b}(x) t_{b c}(x) t_{c a}(x) & =\mathbf{1},
\end{aligned}
$$

for all points $x \in M$ where the functions are all defined. The last condition is the cocycle condition. A gauge transformation is defined to be a collection of maps

$$
\lambda_{a}: U_{a} \rightarrow G .
$$

acting on the transition functions in the following way,

$$
t_{a b}(x) \longmapsto \lambda_{a}^{-1}(x) t_{a b}(x) \lambda_{b}(x) .
$$

Gauge-transformed transition functions define the same bundle. A bundle is characterized by a gauge equivalence classes of transition functions satisfying (61).

A connection $\omega$, defined globally, is represented by a connection one-form $\omega_{a}$ on each $U_{a}$, with values in the Lie algebra of $G$, where, on each overlap $U_{a} \cap U_{b}$,

$$
\omega_{a}=\operatorname{Ad}_{t_{a b}^{-1}} \omega_{b}+t_{a b}^{-1} d t_{a b},
$$

where Ad is the adjoint representation. The curvature two form is $f_{a}=d \omega_{a}+\omega_{a} \wedge \omega_{a}$. The information about the topology of $P(M, G)$, which is coded in the transition functions, can be extracted from integrals of polynomials in the curvature, called characteristic classes, of an arbitrary connection, which represent topological invariants.

In the case where $G=S O(2)$, (64) reduces to (27), the curvature is gauge invariant and therefore is a globally defined two-form $F=f \tau$. If $M$ is a $2 \mathrm{~d}$ connected compact and orientable manifold, the only interesting characteristic class is the first Euler class, whose integral gives the Euler number (31). Since $d f=0$,

$$
e(P)=\frac{1}{2 \pi} \int_{M} f
$$

is an element of the second de Rham cohomology group, $H^{2}(M, \mathbb{Z}) \sim \mathbb{Z}$. The inequivalent principal bundles $P(M, S O(2))$ are therefore classified by the integer $e(P)$. As discussed above, the frame bundle of $M$ is a $P(M, S O(2))$ bundle with $e(P)=\chi(M)$.

Let us consider the sphere $M=S_{2}$ as an example. Let $\theta \in[0, \pi], \phi \in[0,2 \pi]$ be the standard polar coordinates of the sphere. We can choose two open sets, the (slightly extended) northern and the southern hemisphere, $U_{N}$, defined by $\theta \in[0, \pi / 2+\epsilon]$, and $U_{S}$ defined by $\theta \in[\pi / 2-\epsilon, \pi]$. The overlap region is the $2 \epsilon$-wide ribbon around the equator. It is not difficult to see that any transition function is gauge equivalent 
to a transition function of the form $t_{a b}(\theta, \phi)=e^{q \phi \tau}$, where $q$ is an integer. Notice that $q$ counts the number of times the equator wraps around the gauge group. A connection ${ }^{6}$ can be written in the form [22]

$$
\omega_{N}=\frac{q}{2}(1-\cos \theta) d \phi, \quad \omega_{S}=-\frac{q}{2}(1+\cos \theta) d \phi
$$

On the equator, the two one-forms are related by (64), which takes the form $\omega_{N}=\omega_{S}+q d \phi$. The Euler number (65) is

$$
e(P)=\frac{1}{2 \pi} \int_{S^{2}} f=\frac{1}{2 \pi} \int_{\text {equator }}\left[\omega_{N}-\omega_{S}\right]=\frac{1}{2 \pi} \int_{0}^{2 \pi} q d \phi=q .
$$

For $q=0$ we have the trivial bundle $S_{2} \times S O(2)$, which admits the flat connection (66). For $q=1$ we have the principal bundle associated to the Hopf vector bundle (the complex line bundle over the projective plane). Since the Euler number of the sphere is $\chi\left(S^{2}\right)=2$, for $q=2$ we have the frame bundle. To see this, notice that the commonly used dyad $e^{1}=d \theta, e^{2}=\sin \theta d \phi$ is ill-defined at the two poles. We can get a dyad which is well defined at the poles by rotating it in the two hemispheres

$$
\begin{aligned}
e_{N}^{1}=\cos \phi d \theta-\sin \phi \sin \theta d \phi, & & e_{N}^{2}=\sin \phi d \theta+\sin \phi \sin \theta d \phi, \\
e_{S}^{1}=\cos \phi d \theta+\sin \phi \sin \theta d \phi, & & e_{S}^{2}=-\sin \phi d \theta+\sin \phi \sin \theta d \phi .
\end{aligned}
$$

The corresponding connection, that satisfies the Cartan equation (11), is (66) with $q=2$. It is easy to get convinced that if $q$ is different from 2, then the connection (66) does not admit a dyad satisfying (11).

\subsection{Discrete bundles}

Let us now define a bundle over a $2 \mathrm{~d}$ discretized manifold. Consider a $2 \mathrm{~d}$ manifold $M$ and a cellular decomposition $\Delta^{*}$ of $M . \Delta^{*}$ is formed by $2 \mathrm{~d}$ faces $a, b, c, \ldots$, which partition $M$. The faces meet along 1-dimensional edges $e_{a b}$, which, in turn, meet in vertices $v_{a b c}$. (For simplicity we assume here that vertices are trivalent.) Associate to each face $a$ an open subset $U_{a}$ of $M$, containing $a$. Let a bundle be defined by transition functions $t_{a b}$ on the overlaps. Notice that the edge $e_{a b}$ is contained in the overlap $U_{a} \cup U_{b}$. The topological information on the bundle is entirely contained in the restrictions of the transition functions to the edges. Let $s \in[0,1]$ be a coordinate on the edge $e_{a b}$ and let us call $t_{a b}(s)$ this restriction. That is, we consider functions

$$
t_{a b}: e_{a b} \rightarrow G
$$

These transition functions satisfy three conditions:

$$
\begin{aligned}
t_{a a}(s) & =\mathbf{1}, \\
t_{a b}(s) t_{b a}(s) & =\mathbf{1}, \\
t_{a b}(s) t_{b c}(s) t_{c a}(s) & =\mathbf{1},
\end{aligned}
$$

for all points where the functions are all defined. Notice that now the cocycle condition holds at isolated points: the vertices $s=v_{a b c}$. A gauge transformation is a collection of continuous maps from the perimeter of each face $a$ to $G$, such that the image of $\lambda_{a}$ is contractible (because it must be possible to extend it continuously on the entire face.) It acts on the transition functions in the following way,

$$
t_{a b}(s) \longmapsto \lambda_{a}^{-1}(s) t_{a b}(s) \lambda_{b}(s) .
$$

A principal bundle $P\left(\Delta^{*}, G\right)$ is defined by the transition functions (73) up to the gauge transformations (74) ${ }^{7}$ As suggested by (29), the key quantity of interest is the variation of the transition function along each edge. Let us therefore define the quantity

$$
n_{a b}=\frac{1}{2 \pi} \int_{e_{a b}} d s \quad t_{a b}^{-1}(s) \frac{d}{d s} t_{a b}(s)
$$

\footnotetext{
${ }^{6}$ The connection [66 is the one encountered in the Dirac monopole: $q$ is the magnetic charge times the electric charge, and the condition that $q$ is integer is Dirac's quantization of the magnetic charge.

${ }^{7}$ The cohomological structure $d^{2}=0$ determined by $d: \lambda_{a} \longmapsto t_{a b}=\lambda_{a}^{-1} \lambda_{b}$ and $d: t_{a b} \longmapsto \rho_{a b c}=t_{a b} t_{b c} t_{c a}$ is the same as in the continuous case, but restricted to $\Delta^{*}$.
} 
associated to each edge. Restricting to the case $G=S O(2)$ we are interested in, this can be expressed as

$$
n_{a b}=\frac{1}{2 \pi} \int_{e_{a b}} d \psi_{a b}(s),
$$

where $t_{a b}(s)=e^{\psi_{a b}(s) \tau}$. Notice that $n_{a b}=n_{b a}$, because reversing the order of the cells changes the orientation of the edge but also the sign of $\psi_{a b}(s)$, because of (72). A gauge transformation in the open set $a$ adds a real number $\lambda_{a b}$ to each $n_{a b}$. This number is the variation of the gauge transformation along $e_{a b}$, and can be written as $\lambda_{a b}=\lambda_{a}^{i}-\lambda_{a}^{j}$, where $i$ and $j$ label the two vertices bounding $e_{a b}$. The gauge transformation can be written as

$$
n_{a b} \mapsto n_{a b}+\lambda_{a}^{i}-\lambda_{a}^{j}+\lambda_{b}^{j}-\lambda_{b}^{i}
$$

This structure can be simplified using a partial gauge fixing. Because of the cocycle condition (73) it is always possible to choose a gauge transformation (74) such that the transformed $t_{a b}(s)$ is the identity at the vertices, namely $t_{a b}(0)=t_{a b}(1)=1$. Denote this gauge the "edge gauge". Then the cocycle condition is trivially satisfied. In this gauge the transition functions define maps $t_{a b}: S_{1} \rightarrow G$. A gauge transformation deforms these maps smoothly: therefore what is relevant is the homotopy class of these maps. The bundle can be nontrivial only if $\pi_{1}(G)$, the first homotopy group of $G$, is nontrivial. Notice that for $G=S O(2)$ in this gauge (76) is precisely the number of times the transition function of the edge wraps around the group, and is an integer. It is an element of $\pi_{1}(S O(2))$. Within the edge gauge, we can still gauge transform the integers $n_{a b}$ by means of a gauge transformation that is the identity on the vertices, but the quantities $\lambda_{a}^{i}$ in (177) must be integers (more precisely, elements of $\pi_{1}(S O(2))$.)

The bundle is thus uniquely characterized by the discrete set of integers $n_{a b}$ (more precisely, elements of $\left.\pi_{1}(S O(2))\right)$ and the gauge transformations (77). It is clear that the only invariant quantity is

$$
e(P)=\frac{1}{2} \sum_{a b} n_{a b},
$$

which can be recognized as the Euler number of the bundle.

We take the above construction as a motivation, and define the "discrete bundle" $P\left(\Delta^{*}, S O(2)\right)$, namely an $S O(2)$ bundle over an abstract 2 d cellular complex $\Delta^{*}$, by the assignement of an element of $\pi_{1}(S O(2))$ to each edges $e_{a b}$ of $\Delta^{*}$, up to the gauge transformations (177). The extension of this definition to different groups is straightforward. The extension to higher dimensional manifolds will be considered elsewhere.

\section{Quantum $B F$ and GR on an arbitrary topology}

On a nontrivial bundle, we associate a distinct connection one-form $\omega_{a}$ to each open set $U_{a}$. On a cellular manifold, we associate a distinct connection one-form $\omega_{a}$ to each face $a$ (we have assumed that each face is included in an open set.) In discretizing the field theory, we previously considered a single group element (34) associated to each edge $e_{a b}$, separating the face $a$ from the face $b$; this is obtained exponentiating the (scaled) connection. If the bundle is nontrivial, we must consider, instead, two of these group elements, one defined by the connection $\omega_{a}$ and the other defined by the connection $\omega_{b}$. Let us denote them $g_{a b}$ and $g_{b a}$ :

$$
g_{a b}=e^{\frac{k}{\hbar} \int_{e_{a b}} \omega_{a}}, \quad g_{b a}=e^{\frac{k}{\hbar} \int_{e_{b a}} \omega_{b}} .
$$

These two group elements are related by the transition functions. From (27) and (76) we have

$$
g_{a b} g_{b a}=e^{\frac{k}{\hbar} 2 \pi n_{a b} \tau}
$$

which replaces (36) in the general case.

We can now proceed with the quantization precisely as we did for the torus. The only difference is that the relation between $g_{a b}$ and $g_{b a}$ is given by (80) instead than by (36). Consequently, the definition of the measure (44) must be replaced by

$$
d g_{(a b)}=d g_{a b} d g_{b a} \delta\left(g_{a b} g_{b a} e^{-\frac{k}{\hbar} 2 \pi n_{a b} \tau}\right)
$$


Repeating the quantization precisely as in Section 3 we get again to equation (51), but now the relation between $\phi_{a b}$ and $\phi_{b a}$ is determined by (80), namely is

$$
\phi_{a b}+\phi_{b a}=\frac{k}{\hbar} 2 \pi n_{a b}
$$

instead of (37). Therefore (52) is replaced by

$$
Z=\sum_{\left\{n_{a}\right\}} \int \prod_{(a b)} \frac{d \phi_{(a b)}}{2 \pi} e^{\left.i \sum_{(a b)}\left(\left(n_{a}-n_{b}\right) \phi_{a b}+n_{b} \frac{k}{\hbar} 2 \pi n_{a b}\right)\right)} .
$$

performing the integration, and using (78), this gives

$$
Z=\sum_{n} e^{i n \frac{k}{\hbar} 2 \pi \sum_{(a b)} n_{a b}}=\sum_{n} e^{i n \frac{2 \pi k}{\hbar} e(P)} .
$$

In the case of $B F$ theory, we can perform the sum, giving

$$
Z_{B F}=\delta(k / \hbar e(P))
$$

That is, the partition function of $B F$ theory vanishes unless $e(P)=0$, namely unless the bundle is trivial. In a sense, this mean that if the bundle is nontrivial $B F$ theory does not exist. The result can be related to what happens in the classical theory, because on a nontrivial bundle the $B F$ equation (17) has no solution, since there is no flat connection on a nontrivial bundle. If the bundle is trivial, we recover the divergence mentioned above.

In the case of GR, recall that we must: (i) choose the value (24) for the coupling constant $k$; (ii) fix the bundle to be the frame bundle, namely impose (32); and (iii) impose the Plebanski constraint (58). Using this, we have immediately

$$
Z_{\mathrm{GR}}[M]=\sum_{n} \delta_{n^{2}, 1} e^{i n \frac{1}{4 \hbar G} \chi(M)}=2 \cos \left(\frac{\chi(M)}{4 \hbar G}\right) .
$$

This is precisely the result for the partition function of $2 \mathrm{~d}$ riemannian quantum GR, equation (10), as desired. This result has been obtained with a finite calculation, where no divergences or ill-defined quantities appear.

We close this section with a few comments.

First, the same result can be obtained also by directly discretizing and quantizing the action (20). The discretization of (20) can be obtained by adding one term to the discrete action (41)

$$
S_{B F}\left[B_{a}, g_{a b}\right]=-\hbar \sum_{a} \operatorname{tr}\left[B_{a} g_{a}^{1 / 2}-\lambda_{a}\left(B_{a} B_{a}+1\right)\right],
$$

and all the integrals in the definition of the partition function can be easily performed. Here we have preferred to enforce the constraint "by hand" as a constraint on the representations in order to mimic the $4 \mathrm{~d}$ procedure.

Second, to obtain the complex partition function (9), corresponding to a fixed orientation of the spacetime manifold, it is sufficient to restrict the determinant of the dyad to be positive. This restriction of the global orientation is not straightforward in higher dimensions (see [16]), but in this $2 \mathrm{~d}$ case can be easily obtained by replacing the action (20) with

$$
S_{\mathrm{GR}}[B, \omega, \lambda]=-\frac{1}{2} k \int \operatorname{tr}[B F-\lambda(B \tau+\mathbf{1})]=k \int[b f-\lambda(b-1)] .
$$

In this way, the only solution of the Plebanski constraint is $B=+\tau$, i.e. $b=+1$, equivalent to $n=+1$ at the quantum level.

Finally, the reader may wonder what is the meaning or the rationale for the factor $k / \hbar$ that multiplies the gravitational connection in the definition (34) of the group elements used as variables for the discretized theory. At first sight, one may think that this factor can be simply absorbed in the definition of the units in which the connection is measured. Notice that $k / \hbar=1 / 8 \pi G \hbar$ is dimensionless in $2 \mathrm{~d}$. We can certainly 
absorbe this factor in the field by re-scaling $\omega$ and defining $\tilde{\omega}=(k / \hbar) \omega$. However, if we multiply a connection by a real number, we do not obtain a connection. More precisely, if we insist that $\tilde{\omega}$ be a connection, we have to modify the gauge trasformations, and thefore, ultimately, change the "size" of the gauge group, which shows up in (76). In other words, the quantum theory does depend on a dimensionless number which expresses precisely the ratio between the dimension of the phase defined by the action and the dimension of the gauge group.

\section{Summary and perspectives}

Riemannian general relativity in $2 \mathrm{~d}$ can be written as a $B F$ theory plus a Plebanski-type constraint. This is analogous to what happens in $4 \mathrm{~d}$. The quantization can be performed imposing the constraint in the spinfoam theory as a restriction of the representations summed over. The partition function of $B F$ theory diverges on a trivial bundle and vanishes on nontrivial bundles. The partition function of GR, on the other hand, is well-defined for all topologies of the spacetime manifold, there are no divergences, and agrees with the one obtained from formal manipulations of the functional integral. This result can be taken as indirect support to the viability of similar techniques used in $4 \mathrm{~d}$ quantum gravity.

Care should be taken concerning the bundle topology. The topology of the bundle on which the gravitational connection is defined is the frame bundle. It is determined by the topology of spacetime and in general it is nontrivial. In the discretization of the continuous theory, we need to take the topology of the bundle into account. This topology can be coded by integers (elements of $\pi_{1}(S O(2))$ ) associated to the edges of the cellular complex used in the discretization. These data define a nontrivial $S O(2)$ principal bundle over a simplicial complex. The spinfoam theory must be defined on this nontrivial discrete bundle.

We close with some general considerations on possible developments. Bundle topology, and higher dimensional homotopy groups, might play a role also in higher dimensions. It may be possible to refine the higher dimensional spinfoam models introducing the additional data encoding the fact that we are dealing with a connection on the frame bundle, which is topologically non-trivial for non-trivial spacetime manifolds. In the $3 \mathrm{~d}$ case we expect the $\pi_{2}(G)$ homotopy group to provide the relevant data. Notice that nontriviality appears only if we use $S O(3)$, since any $S U(2)$ bundle in $3 \mathrm{~d}$ is trivial.

In $4 \mathrm{~d}$, it is reasonable to expect that the relevant homotopy group is $\pi_{3}(S O(4))$. In the case, the topological data entering the action are likely to be insufficient to characterize the frame bundle. But a detailed analysis is needed before drawing a conclusion. In 4d, GR has local degrees of freedom, and global issues may be of lesser importance. However, when the definition of the theory includes a sum over topologies, topological dependent factors might sensibly affect the sum. Notice for example that spacetime topology is not fixed in the group field theory (GFT) approach to the spinfoam formalism; see [1, 2].

If quantum gravity is formally defined as a path integral for the gravitational connection field $\omega$, one might also consider the possibility of treating the topology of the bundle as a variable in the quantum theory, and of summing over bundle topologies in a sum-over-histories formulation. Here, we have considered the bundle topology fixed to the value imposed by the Cartan equation (11). In the GFT formalism described in the appendix, on the other hand, it appears that the quantities encoding the bundle topology can only be added as dynamical variables to be summed over.

Finally, we have not discussed transition amplitudes. In a topological quantum field theory, these can be obtained by computing the partition functions with fixed boundary conditions 24. The Euler characteristic of a $2 \mathrm{~d}$ compact manifold with $b$ boundaries is given by $\chi(M)=2-2 g-b$. We expect that the setting discussed here could be extended to the case with boundaries, with boundary data in the $n=1$ representation of $S O(2)$, and transition amplitudes weighted by $e^{\frac{i}{4 \hbar G} \chi(M)}$. For a manifold with genus $g$ and $b$ boundaries, the only nontrivial transition function should have the structure

$$
W_{\mathrm{GR}} \sim e^{\frac{4 \pi i}{\hbar G}(2-2 g-b)} .
$$

We will discuss these amplitudes, together with the GFT that generates them, elsewhere.

Among other issues that have not yet been studied in this context, we signal the lorentzian theory, matter and particle couplings (see [21, 27]).

In spite of its simplicity $2 \mathrm{~d}$ gravity proves to be a model where there is still much to learn.

\section{Acknowledgments}



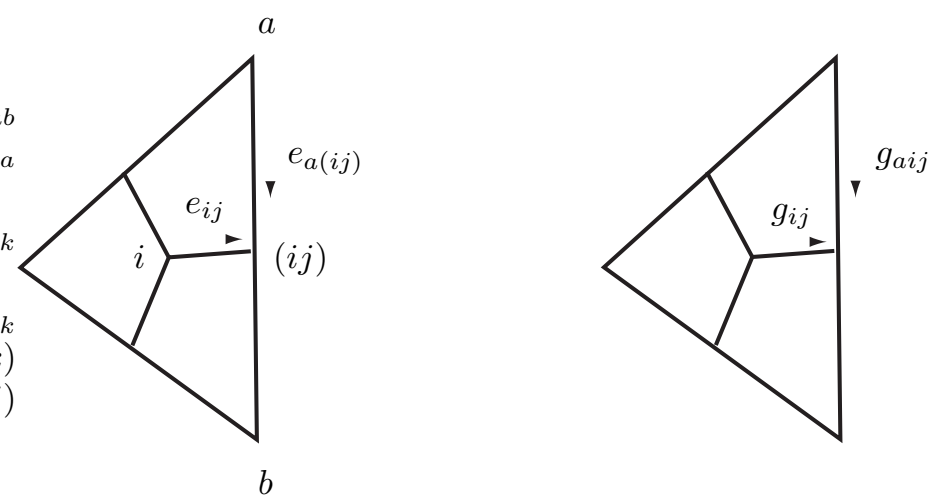

Figure 2: A triangle $i$ of the triangulation $\Delta$, divided in three wedges. The left panel indicates the names of the segments. The right panel indicates the group elements associated to these segments.

Thanks to Dario Benedetti, Matyas Karadi and Etera Livine for useful discussions. Thanks to Andrea Sambusetti for many clarifications on principal bundles and the Gauss-Bonnet theorem. SS thanks Rome's Physics Department and INFN's group TS11 for support.

\section{Appendix}

Here we describe a second version of the construction of a discrete bundle. In Section 4.2 we assumed that each face of the cellular complex $\Delta^{*}$ was contained in a single chart. Here we assume that each triangle of the triangulation $\Delta$ is contained in a single chart. This construction is equivalent but slightly more complicated than the one given in 4.2 it has the advantage that it matches very naturally the GFT formalism. Furthermore, it should be more adapted to higher dimensions. Only in $2 \mathrm{~d}$ the faces of $\Delta^{*}$ have the same dimension as spacetime. In $n$ dimensions, we can relate the $n$-simplices of the simplicial decomposition $\Delta$ of the manifold with the ( $n$-dimensional) open sets where the local charts are defined.

Assume that each triangle $i, j, k, \ldots$ of a triangulation $\Delta$ lies within a single chart. Denote $s_{i j}$ the oriented segment separating the triangles $i$ and $j$. Consider the restriction of the transition functions to these segments. This restriction defines the transition functions

$$
t_{i j}: s_{i j} \rightarrow G
$$

that satisfy conditions analogous to (71), (72), (73). Denote $p_{a}$ and $p_{b}$ the end points of the segment $s_{i j}$. These are dual to the faces $a$ and $b$ of $\Delta^{*}$. Partition each segment $s_{i j}$ into two parts, by choosing an arbitrary point $p_{(i j)}$ on it. Call $e_{a(i j)}$ the oriented segment going from $a$ to $p_{(i j)}$. Thus the segment $s_{i j}$ is the composition of $e_{a(i j)}$ and $e_{b(i j)}^{-1}$. See Figure 2 .

A gauge transformation is a collection of maps $\lambda_{i}$ from the perimeter of each triangle $i$ to $G$, such that its image of the perimeter is contractible (because it must be possible to extend the map continuously to the entire triangle). It acts on the transition functions in the following way,

$$
t_{i j}(s) \longmapsto \lambda_{i}^{-1}(s) t_{i j}(s) \lambda_{j}(s) .
$$

A principal bundle is defined by the transition functions (90) up to these gauge transformations.

As before, the key quantity of interest is the variation of the transition function along each half-segment. Let us therefore define the quantity

$$
n_{a(i j)}=\frac{1}{2 \pi} \int_{e_{a(i j)}} d s \quad t_{i j}^{-1}(s) \frac{d}{d s} t_{i j}(s)
$$

associated to each edge. Restricting to the $G=S O(2)$ case we are interested in, this can be expressed as

$$
n_{a(i j)}=\frac{1}{2 \pi} \int_{e_{a(i j)}} d \psi_{a(i j)}(s),
$$


where $t_{a(i j)}(s)=e^{\psi_{a(i j)}(s) \tau}$. We also define

$$
n_{a b}=n_{a(i j)}+n_{b(i j)}
$$

A gauge transformation in the open set $i$ can add an arbitrary real number $\lambda_{a(i j)}$ to each $n_{a(i j)}$. This number is the variation of the gauge transformation along $e_{a(i j)}$. The gauge transformation can be written as

$$
n_{a(i j)} \mapsto n_{a(i j)}+\lambda_{i}^{a}-\lambda_{i}^{j}-\lambda_{j}^{a}+\lambda_{j}^{i} .
$$

As before, this structure can be simplified using a partial gauge fixing, which makes it much easier to incorporate the cocycle condition directly into the quantities $n_{a(i j)}$. Because of the cocycle condition (73) it is always possible to chose a gauge transformation (91) such that the transformed $t_{i j}(s)$ is the identity at the vertices, as well as on the points $p_{(i j)}$. Denote this gauge the "edge gauge". Then the cocycle condition is trivially satisfied. In this gauge the transition functions define maps $t_{a(i j)}: S_{1} \rightarrow G$ and (93) is the number of times the transition function of the segment wraps around the group, and is an integer. Within this gauge, we can still gauge transform the integers $n_{a(i j)}$ by means of a gauge transformation that is the identity on the vertices, but the quantities $\lambda_{i}^{a}$ and $\lambda_{i}^{j}$ and in (95) must be integers.

The bundle is uniquely characterized by the discrete set of integers numbers $n_{a(i j)}$, and the gauge transformations (95). The only invariant quantity is

$$
e(P)=\frac{1}{2} \sum_{a(i j)} n_{a(i j)},
$$

which can be recognized again as the Euler number of the principal bundle.

In order to write the discretization of the field theory, we use the "derived complex" 25. Its utility for the spinfoam formalism was recognized by Reisenberger in [26]. This is obtained by considering both a triangulation $\Delta$ of the manifold and its dual cellular complex $\Delta^{*}$. Denote $v_{i}=v_{(a b c)}$ a vertex of the dual complex $\Delta^{*}$ (an arbitrary point inside the triangle $i$.) Let $e_{i j}$ be a segment joining the point $v_{i}$ with the point $p_{(i j)}$. Notice that the edges $e_{a b}$ of the dual complex $\Delta^{*}$ are formed by the conjunctions of $e_{i j}$ and $e_{j i}^{-1}$. The intersection of the triangle $i$ (of $\Delta$ ) and the face $a$ (of $\Delta^{*}$ ) is a quadrangle called a wedge and denoted $w_{a i}$. It is bounded by the four segments $e_{i j}, e_{a(i j)}^{-1}, e_{a(i k)}$ and $e_{i k}$. See Figure 2 and Figure 3.

We now introduce a discretization of the connection field $\omega$. This will be given by two kinds of group elements. First, the group elements $g_{i j}$ associated with the oriented segments $e_{i j}$

$$
g_{i j}=e^{\frac{k}{\hbar} \int_{e_{i j}} \omega_{i}}
$$

where $\omega_{i}$ is the one-form representing the connection in the open set to which the triangle $i$ belongs. Second, the two group elements $g_{a i j}$ and $g_{a j i}$, both associated with the segment $e_{a(i j)}$, and interpreted as the exponential of the scaled connection in the two open sets $i$ and $j$ respectively.

$$
g_{a i j}=e^{\frac{k}{\hbar} \int_{s_{a(i j)}} \omega_{i}}, \quad g_{a j i}=e^{\frac{k}{\hbar} \int_{s_{a(i j)}} \omega_{j}} .
$$

These two group elements are related by the transition functions

$$
g_{a i j}\left(g_{a j i}\right)^{-1}=e^{\frac{k}{\hbar} 2 \pi n_{a(i j)} \tau} .
$$

The discretized action can be written as sum over the wedges $w_{a i}$. We define the (exponentiated) curvature associated to each wedge $a$ as the product of the group elements associated to the segments forming its perimeter. See Figure 3

$$
g_{a i}=g_{a i j} g_{i j}^{-1} g_{i k} g_{a k i}^{-1}
$$

The dynamics of the discrete model is defined by discretizing the action (15) as

$$
S_{B F}\left[B_{a}, g_{i j}, g_{a i j}\right]=-\hbar \sum_{a i} \operatorname{tr}\left[B_{a} g_{a i}^{1 / 2}\right]
$$

This defines the discretized model in the second version. Notice that this sum can be entirely rewritten in terms of the group elements

$$
g_{a b}=g_{i j} g_{a i j}^{-1} g_{a j i} g_{j i}^{-1}
$$




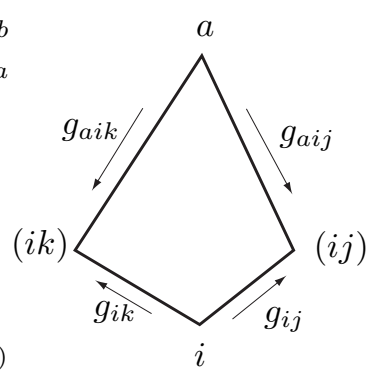

Figure 3: A wedge $w_{a i}$ and the group elements on its perimeter.

which can be identified as the group elements denoted in the same manner in section 4.2 The relation between $g_{a b}$ and $g_{b a}$ follows from (99) and turns out to be precisely given by (80), where $n_{a b}$ is now given by (94). This shows the equivalence with the previous version.

The group elements $g_{i j}$ and $g_{a i j}$ can be included in the GFT formulation as follows (see 12.) A 2d GFT [13] is defined by a field $\phi\left(g_{1}, g_{2}\right)$ on $S O(2) \times S O(2)$, and by the action

$$
S[\phi]=\int d g_{1} d g_{2} \phi\left(g_{1}, g_{2}\right) \phi\left(g_{1}, g_{2}\right)+\lambda \int d g_{1} d g_{2} d g_{3} d g d g^{\prime} d g^{\prime \prime} \phi\left(g_{1} g, g_{2} g\right) \phi\left(g_{2} g^{\prime}, g_{3} g^{\prime}\right) \phi\left(g_{3} g^{\prime \prime}, g_{1} g^{\prime \prime}\right)
$$

(for convenience we have inserted the projection implementing gauge invariance in the interaction term instead than in the kinetic term.) The Feynman expansion can be written in terms of the propagator

$$
K\left(g_{1}, g_{2} ; g_{1}^{\prime}, g_{2}^{\prime}\right)=\delta\left(g_{1}, g_{1}^{\prime}\right) \delta\left(g_{2}, g_{2}^{\prime}\right),
$$

and the vertex

$$
V\left(g_{1}, g_{2} ; g_{1}^{\prime}, g_{2}^{\prime} ; g_{1}^{\prime \prime}, g_{2}^{\prime \prime}\right)=\lambda \int d g d g^{\prime} d g^{\prime \prime} \delta\left(g_{1} g, g_{1}^{\prime} g^{\prime \prime}\right) \delta\left(g_{2} g, g_{2}^{\prime} g^{\prime}\right) \delta\left(g_{3} g^{\prime}, g_{3}^{\prime} g^{\prime \prime}\right) .
$$

The Feynman combinatorics gives a sum over two-complexes because sequences of delta functions with corresponding arguments define loops on the Feynman graph that can be identified with faces [11. Let us call $i$ the vertices and $a$ the faces of one two-complex. Then the group element appearing in the propagator connecting the vertex $i$ with the vertex $j$ and belonging to the face $a$ can be called $g_{a i j}$. The group elements appearing in the integrals in the vertex and belonging to end of the vertex $i$ that gets connected with the vertex $j$ can be called $g_{i j}$. It is then a simple exercise to see that the weight of a two-complex is given by the partition function of the spinfoam model considered above.

To take into account the possibility of a nontrivial bundle, in the GFT formalism we can modify the kinetic term. In fact, imagine to replace the kinetic term in (103) as follows

$$
S[\phi]=\int d g_{1} d g_{2} \phi\left(g_{1} h, g_{2} h^{\prime}\right) \phi\left(g_{1}, g_{2}\right)+\lambda \int d g_{1} d g_{2} d g_{3} d g d g^{\prime} d g^{\prime \prime} \phi\left(g_{1} g, g_{2} g\right) \phi\left(g_{2} g^{\prime}, g_{3} g^{\prime}\right) \phi\left(g_{3} g^{\prime \prime}, g_{1} g^{\prime \prime}\right) .
$$

where

$$
h=e^{\frac{k}{\hbar} 2 \pi n \tau}, \quad h^{\prime}=e^{\frac{k}{\hbar} 2 \pi n^{\prime} \tau} .
$$

Then the propagator becomes

$$
K\left(g_{1}, g_{2} ; g_{1}^{\prime}, g_{2}^{\prime}\right)=\delta\left(g_{1} h, g_{1}^{\prime}\right) \delta\left(g_{2} h^{\prime}, g_{2}^{\prime}\right) .
$$

This modifies the spinfoam model simply by replacing

$$
\delta\left(g_{a i j}, g_{a j i}\right)
$$

with

$$
\delta\left(g_{a i j} h, g_{a j i}\right) .
$$


That is, it implements (99), where $h$ can be identified with $e^{\frac{k}{\hbar} 2 \pi n_{a(i j)} \tau}$. However, such a modification would give all transition function equal to one another. To have different transition functions on different segments we have to consider the transition functions as dynamical variables and sum over them. Thus, introducing the bundle topology in the GFT appears to require a sum over the topologies of the fiber bundles. This can be achieved by considering the action

$$
\begin{aligned}
S[\phi]=\sum_{n, n^{\prime}} c_{n} c_{n^{\prime}} & \int d g_{1} d g_{2} \phi\left(g_{1} e^{\frac{k}{\hbar} 2 \pi n \tau}, g_{2} e^{\frac{k}{\hbar} 2 \pi n^{\prime} \tau}\right) \phi\left(g_{1}, g_{2}\right) \\
& +\lambda \int d g_{1} d g_{2} d g_{3} d g d g^{\prime} d g^{\prime \prime} \phi\left(g_{1} g, g_{2} g\right) \phi\left(g_{2} g^{\prime}, g_{3} g^{\prime}\right) \phi\left(g_{3} g^{\prime \prime}, g_{1} g^{\prime \prime}\right),
\end{aligned}
$$

for suitable weights $c_{n}$. The Feynman expansion of this action gives a spinfoam model that involves a sum over (topologically inequivalent) two-complexes as well as over all fiber bundle topologies. The properties of this sum will be studied elsewhere.

\section{References}

[1] D. Oriti. "Spacetime geometry from algebra: spinfoam models for non-perturbative quantum gravity". Rept. Prog. Phys. 64, 1489, 2001; gr-qc/0106091

[2] A. Perez. "Spinfoam models for quantum gravity". Class. Quant. Grav., 20:R43, 2003; gr-qc/0301113

[3] C. Rovelli. Quantum Gravity. (Cambridge University Press, Cambridge 2004).

[4] J. W. Barrett and L. Crane. "Relativistic spin networks and quantum gravity". J. Math. Phys., 39:3296-3302, 1998; gr-qc/9709028

[5] A. Perez, C. Rovelli, "A spinfoam model without bubble divergences". Nucl Phys B599, 255-282, 2001.

[6] A. Perez, "Finiteness of a spinfoam model for euclidean GR". Nucl Phys B599, 427-434, 2001.

[7] L. Crane, A. Perez and C. Rovelli, "Perturbative Finiteness in Spin-Foam Quantum Gravity". Phys Rev Lett 87, 181301, 2001. A Finiteness Proof for the Lorentzian State Sum Spin Foam Model for Quantum General Relativity, gr-qc/0104057.

[8] T. Thiemann, Modern Canonical Quantum General Relativity (Cambridge University Press, Cambridge, to appear in 2004), a preliminary version is in gr-qc/0110034.

[9] J. Ambjorn, B. Durhuus and T. Jonsson. Quantum Geometry. (Cambridge University Press, Cambridge 1997.)

[10] M. Blau and G. Thompson. "Lectures on 2-d gauge theories: Topological aspects and path integral techniques", in Proceedings of the 1993 Trieste Summer School on High Energy Physics and Cosmology, E. Gava et al. eds; pg. 175-244. (World Scientific, Singapore 1994.) hep-th/9310144

[11] R. DePietri, L. Freidel, K. Krasnov and C. Rovelli. "Barret-Crane model from a Boulatov-Ooguri field theory over a homogeneous space", Nucl. Phys. B 574, 785-806, 2000; hep-th/9907154.

[12] M. Reisenberger and C. Rovelli. "Spacetime as a Feynman diagram: the connection formulation". Class.Quant.Grav. 18, 121-140, 2001; gr-qc/0002095

[13] E. R. Livine, A. Perez, and C. Rovelli. "2d manifold-independent spinfoam theory". Class. Quant. Grav., 20:4425-4445, 2003; gr-qc/0102051

[14] G. Ponzano, T. Regge. "Semiclassical limit of Rcah coefficients", in Spectroscopy and group theoretical methods in Physics, F. Bloch ed. (North-Holland, Amsterdam, 1968).

[15] S. S. Chern, W. H. Chen, and K. S. Lam. Lectures on Differential Geometry. (World Scientific, Singapore 1999.) 
[16] E. R. Livine and D. Oriti. "Implementing causality in the spin foam quantum geometry". Nucl. Phys. B, 663:231-279, 2003; gr-qc/0210064

[17] R. De Pietri and L. Freidel. "so(4) Plebanski action and relativistic spinfoam model". Class. Quant. Grav., 16:2187-2196, 1999; gr-qc/9804071.

[18] M. Reisenberger, "Classical Euclidean GR from left handed area = right-handed area". Class and Quantum Grav, 16 1357-1371, 1999; gr-qc/9804061

[19] T. Regge. "General relativity without coordinates". Nuovo Cimento, 19:558-571, 1961.

[20] L. Freidel and D. Louapre. "Diffeomorphisms and spinfoam models". Nucl. Phys. B, 662:279-298, 2003; gr-qc/0212001

[21] L. Freidel and D. Louapre. "Ponzano-Regge model revisited I: Gauge fixing, observables and interacting spinning particles". hep-th/0401076

[22] M. Nakahara. Geometry, Topology and Physics. (IOP Publishing, Bristol 1990).

[23] C. Nash and S. Sen, Topology And Geometry For Physicists. Academic Press, 1983.

[24] M. Atiyah. The Geometry and Physics of Knots. (Cambridge University Press, Cambridge 1990.)

[25] C. F.. Maunder. Algebraic Topology. (Dover, New York 1997.)

[26] M. P. Reisenberger. "A left-handed simplicial action for euclidean general relativity". Class. Quant. Grav., 14:1753-1770, 1997; gr-qc/9609002

[27] K. Noui and A. Perez. "Observability and Geometry in Three dimensional quantum gravity". gr-qc/0402113 